\title{
SYNTHESIS, CHARACTERIZATION AND ANTIMICROBIAL EVALUATION OF SOMETHIAZOLIDINONEDERIVATIVES BEARING PYRIDINE MOIETY: SEARCH FOR ANTIMIROBIAL AGENT
}

\author{
ALI. KHALIL** , RAAFAT A. ELISWAY*, H.A.EYADA*, HAZEM.A. MOHAMED*, \\ GAMEEL A. M. EL-HAG ALI* \\ Departments of Chemistry*, Faculty of Science, Al-Azhar University, Nasr City, Cairo(boys) Egypt \\ Chemistry**, Faculty of Science, Ain Shams University, Cairo, Egypt
}

\begin{abstract}
:
2-(4-Oxothiazolidin-2-ylidene)acetonitrile and ethyl-2-(4-oxothiazolidin-2-ylidene)acetate (1a,b),were condensed with anthralaldehyde(1:1 molar ratio) and gave 4,5-dihydro-4-oxothiazole derivatives (2a,b).Refluxingof 2-(antharacene9-yl-methylene) malononitrile in acetic acid with thioglycollic acid gave(3).4-Thiaz-olidinones containing bis-aryl methylidine moieties $(4 a-c)$ and $(5 a, b)$ were produced via condensation of either(2a) or (3) with aromatic aldehydes(1:1 molar ratio). Heating of (2a) with $\alpha$-substituted cinnamonitriles gave the expected substituted thiazolo[3,2-a] pyridines (6a-d),(7) and (9a-c). Thiazolo [3,2-a] pyridine enamino-nitrile (6a) was refluxed with formic acid and phenyl hydrazine to form the corres-ponding thiazolo [3,2-a] pyridine derivatives (10) and (11) derivatives respectively. The structures of the prepared compounds were confirmed by using spectroscopic techniques; IR; ${ }^{1} H N M R,{ }^{13} \mathrm{C} N M R$ and Mass spectroscopy. Also, Anibactreial and antifungal activity were evaluated for some of synthesized compounds.
\end{abstract}

Keywords; 4-Thiazolidinones, thiazolo [3,2-a] pyridines, and anthracene.

\section{INTRODUCTION}

The increasing cases of microbial resistance pose a major concern to the scientific community and have become a threat for human life worldwide. Moreover, invasive microbial infections caused by multi-drug-resistant Grampositive bacteria and microbes are difficult to diagnose and treat [1]. They are the major cause of morbidity andmortality especially in immune suppressed and hospital-acquiredpatients.To overcome these problems, the development of new and safe antimicrobial agents withbetter effectiveness is urgently required.

To this end, one of the best ways to design new antimicrobial agents is to generate hybrid molecules by combining two bioactive heterocyclic moieties in a single molecular scaffold.Thiazoles are synthetic intermediates and common substructures in numerous biologically active compounds [2-5].There has been considerable interest in the chemistry of thiazolidin-4-one ring system, which is a core structure in various synthetic pharmaceuticals displaying a broad spectrum of biological activities [6-8] and exhibits highly specific activity in vitro against Mycobacterium tuberculosis [9-12]. Furthermore, the pyridine scaffold is a wide spread structural motif that can be found in many natural products and in several pharmacologically interesting compounds. Therefore the synthesis of pyridine derivatives, aiming to develop new drugs, is an active research area. Recently several researchers becameinterested to cyanopyridine derivatives [13-21]. It is thought of interest to accommodate thiazolidin-4-one and pyridine moiety in a single molecular framework and screen for their antimicrobial activity. Motivated by these findings and in continuation of our ongoing efforts on the synthesis of heterocycles with potential antimicrobial activities [22-28], we are purposed to synthesize and investigate the antimicrobial activity of a new series from thiazolidinones class having pyridine moiety.

\section{RESULTS AND DISCUSSION}

The synthesis of the target compounds is depicted in Schemes 1-4. Compound (1)[29] is characterized by the presence of two active methylene as well as nitrile and carbonyl groups which make it chemically very active so it can be used as a precursor to synthesize many biologically and chemically active ring systems. Condensation of acetonitrile (1a) with 9-anthranldehyde (1:1 molar ratio) in ethanol containing catalytic amount of piperidineafforded 
the arylidene derivative as a single product for which (2a) or (3)seemed possible. Compound (3) has been synthesized only through another route via the reaction of 2-(anthracen-9-ylmethylene) malononitrile with thioglycolic acid. This, prove that, anthralaldehyde condensed first with for 4-thiazolidinone ring (1a) at the most active methylene at position number 5rather than exocyclic methylene at position 3 (Scheme 1). Similarly ethyl 5-(anthracen-9-ylmethylene)4-oxothiazolidin-2-ylidene) acetate (2b) was synthesized through condensation of(1b)with anthraldehyde in refluxing ethanol containing few drops of piperidine, (Scheme 1). The structures of the latter products were confirmed by spectroscopic studies and elemental analysis. The IR spectrum of compound (2a), for example, revealed absorption bands at 3183, 2195 and $1707 \mathrm{~cm}-1$ corresponding to an $\mathrm{NH}$, cyano and carbonyl characteristics for thiazolidinone. Its ${ }^{1} \mathrm{H}-\mathrm{NMR}$ spectrum revealed signalsat $\delta 5.16$ and 11.49ppmdue to methine- $\mathrm{H}$ and $\mathrm{NH}$ protons. Whereas, ${ }^{1} \mathrm{H}-\mathrm{NMR}$ spectrum of (2b) revealedbesidesthe specific signals for methine and $\mathrm{NH}$ at 5.57 and 11.49, there are two signals at 1.08 as triplet and at $3.96 \mathrm{ppm}$ as quartet

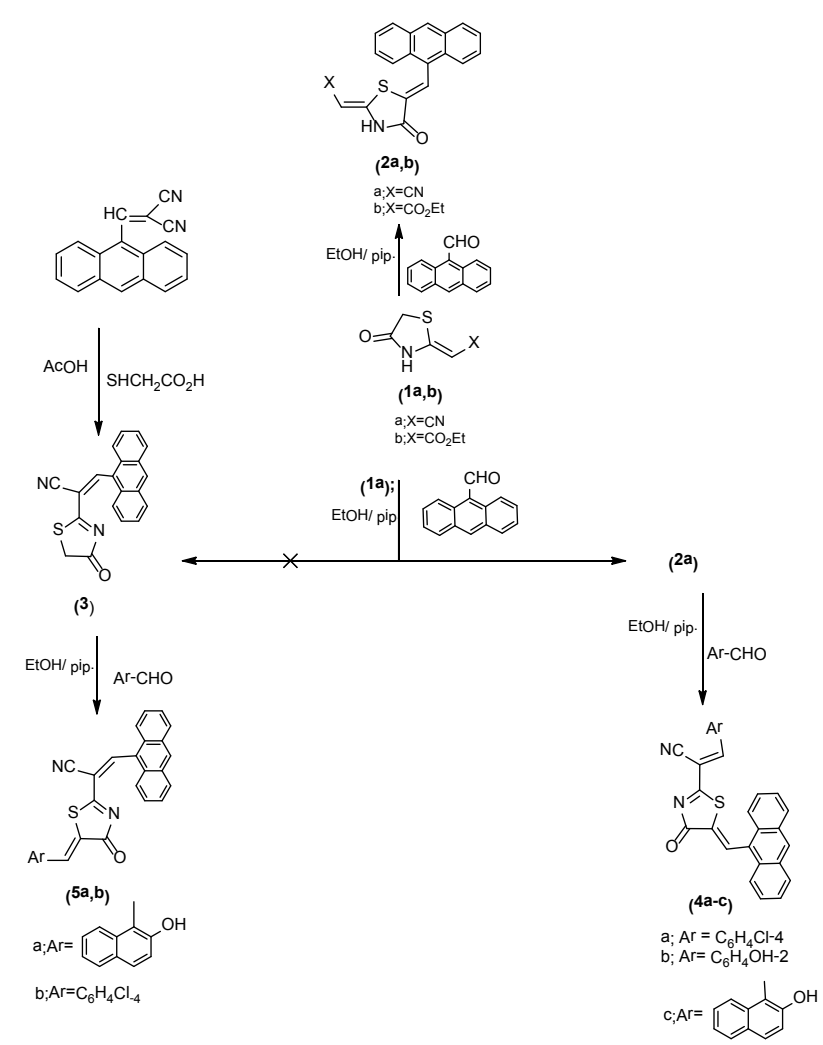

Scheme1 corresponding for the ester group. IR spectrum of (3) assigned stretching absorption band at 1653 $\mathrm{cm}^{-1}$ assignable to ethyleneic carbonyl group. The key starting material (2a) is characterized by the presence of active methylene as well as nitrile groups which make it chemically very active so it can be used as a precursor to synthesize many biologically and chemically active ring systems. Condensation of (2a) with various aromatic aldehydes (1:1 molar ratio) in ethanol catalytic with piperidine afforded the arylidene derivatives (4a-c). Similarly the arylidene derivatives $(5 a, b)$ were obtained through condensation of compound (3) with different aromatic aldehydes (1:1 molar ratio) under reflux conditions. The spectral data of the isolated products were in complete agreement with structures(4) and (5). The IR spectrum for compound (4b) as example revealed absorption bands at 3330, 2204 and $1655 \mathrm{~cm}^{-1}$ corresponding to $\mathrm{OH}, \mathrm{C} \equiv \mathrm{N}$ and $\mathrm{C}=\mathrm{O}$ function respectively. The ${ }^{1} \mathrm{H}-\mathrm{NMR}$ spectrum of (4a) (DMSO-d6) showed multiplet signal at $\delta$ $6.58-8.66$ region distinctive for aromatic protons beside two singlet signals at 9.01, 9.05ppm characteristic of two methine protons. The mass spectrum of (4b) showed a molecular ion peak at $\mathrm{m} / \mathrm{z}=432(0.76 \%)$, corresponding to molecular formula $\mathrm{C}_{27} \mathrm{H}_{16} \mathrm{~N}_{2} \mathrm{O}_{2} \mathrm{~S}$.

In view of the growing biological importance of fused cyanopyridones, particularly thiazolo [1,2-a] pyridines [30-32], it was of interest to synthesize some thiazolo- pyridine derivatives containing anthrancenyl moiety on the hope of obtaining more active compounds. Thus, treatment of $(2 a)$ with $\alpha$-cyanocinnamonitriles under reflux conditions in ethanol furnished a single product for which structure (6) was considered (Scheme 2). Both elemental analysis and spectral data of the isolated products were in assignment with the obtained product. The IR spectrum of (6a)as an example showed the presence of absorption bands 3375,3354, 2200 and 1708 corresponding for $\mathrm{NH}_{2}, \mathrm{C} \equiv \mathrm{N}$ and $\mathrm{C}=\mathrm{O}$ functions respectively. Its ${ }^{1} \mathrm{H}$ NMR spectrum exhibited the lack of singlt signal at $\delta 5.16$ specific for the methine proton and the presence a singlet signal at 4.70 due to pyridine- $4 \mathrm{H}$. The mass spectrum showed a molecular ion peak at $\mathrm{m} / \mathrm{z}=516$ corre- 
sponding to a molecular formula $\mathrm{C}_{30} \mathrm{H}_{17} \mathrm{C}_{1} \mathrm{~N}_{4} \mathrm{O}_{\mathrm{S}}$. Chemically, the structure of (6a) was also confirmed via the reaction of $(4 \mathrm{a})$ with one mole of malononotrile under reflux conditions (mp. an mixed $\mathrm{mp}$, and IR spectra). The dihydropyrano [2',3': 4,5 ] thiazolo[3,2-a] pyridine(7) was achieved as a sole product through one pot reaction of (2a) with p-hydroxybenzaldehyde and malononitrile (1:1:2 molar ratio) (Scheme 2). Actually, the product of this reaction was elucidated on the basis of its spectral dat. The IRspectrum has no absorption band characteristic to a carbonyl group and it revealed the presence of characteristic absorption bands at 3316,3206 and 2201 due to $\mathrm{NH}_{2}$ and $\mathrm{C} \equiv \mathrm{N}$, respectively. ${ }^{1} \mathrm{H}-$ NMR spectrum of (7) supported its structure, as it revealed the 4-Hpyrane and 4H-pyridine ring protons as two singlet signals at $\delta 4.14$ and 4.34 ppm respectively beside the other expected signals.Its mass spectrum showed the molecular ion at $\mathrm{m} / \mathrm{z}=564$ (41.47) corresponding to a molecular formula $\mathrm{C}_{33} \mathrm{H}_{20} \mathrm{~N}_{6} \mathrm{O}_{2} \mathrm{~S}$.

The foregoing results prompted us to investigate the applicability and synthetic potency of (2)to develop a facile and convenient route to thiazolopyridinesof an expected pharmaceutical interest.Thus, reaction of( $2 a)$ with $\alpha$-ethoxycarbonylcinnamonnitriles in refluxing ethanol containing piperidine gave a product for which (8) and (9)can be formulated.On the basis of ana- lytical and spectral data structure (8)was readily eliminated. The structure of (9) was confirmed based on the elemental analyses and spectral data. Thus, the IR spectrum of (9a) as example showed the presence bands at3414, 3289, 2210 and 1714 corresponding for $\mathrm{NH}_{2}, \mathrm{C} \equiv \mathrm{N}$ and $\mathrm{C}=\mathrm{O}$ ester. Its ${ }^{1} \mathrm{H}$ NMR spectrum exhibited triplet signal at $0.83 \mathrm{~J}=7.5 \mathrm{~Hz}$ and quartet signal at 3.88 $\mathrm{J}=7.5 \mathrm{~Hz}$ corresponding for the ethoxy ester function beside singlet signal at $5.16 \mathrm{ppm}$ due to pyridine- $\mathrm{H}$. The mechanism of the reaction was proceeds via generation of carbanion of $\mathrm{CH} 2$ which attack $\beta$-carbon of cinnamonitriles followed by proton shift (Scheme3).

In addition, enaminonitrile(6a)was found to react with either formic acid or phenylhydrazine and the corresponding pyrimidine or pyrazole derivatives were obtained(Scheme4). The structures of isolated products (10) and (11) were confirmed by correct elemental and spectral data. IR spectrum of compound(11)appeared lack of stretching absorption band for carbonyl group. Mass spectrum of (11) assigned a molecular ion peak at $\left(604\left(\mathrm{M}^{+}\right) ; 44.36 \%\right)$ corresponding with molecular formula $\mathrm{C}_{36} \mathrm{H}_{21} \mathrm{C}_{1} \mathrm{~N}_{6} \mathrm{~S}$.

\section{ANTIMICROBIAL ACTIVITY}

\section{Biological evaluation}

Twelve of the synthesized target compounds were tested for in vitro antibacterial activity against three Gram-positive bacteria, Staphylo-
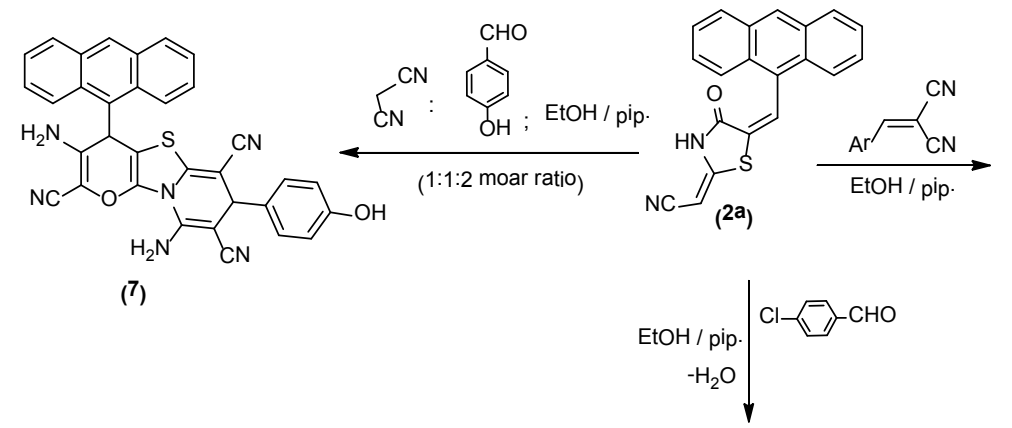

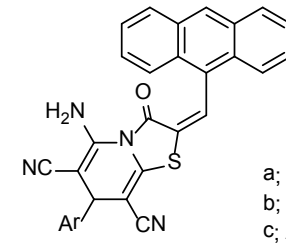

(6b-d)
; $\mathrm{Ar}=\mathrm{C}_{6} \mathrm{H}_{4} \mathrm{Cl}-4$

b; $\mathrm{Ar}=\mathrm{C}_{6} \mathrm{H}_{4} \mathrm{Cl}-2$

$\mathrm{d} ; \mathrm{Ar}=$
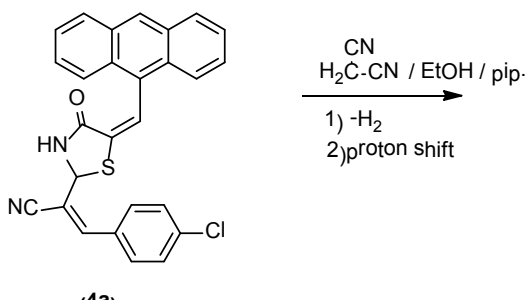

(6a)

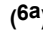

Scheme2 


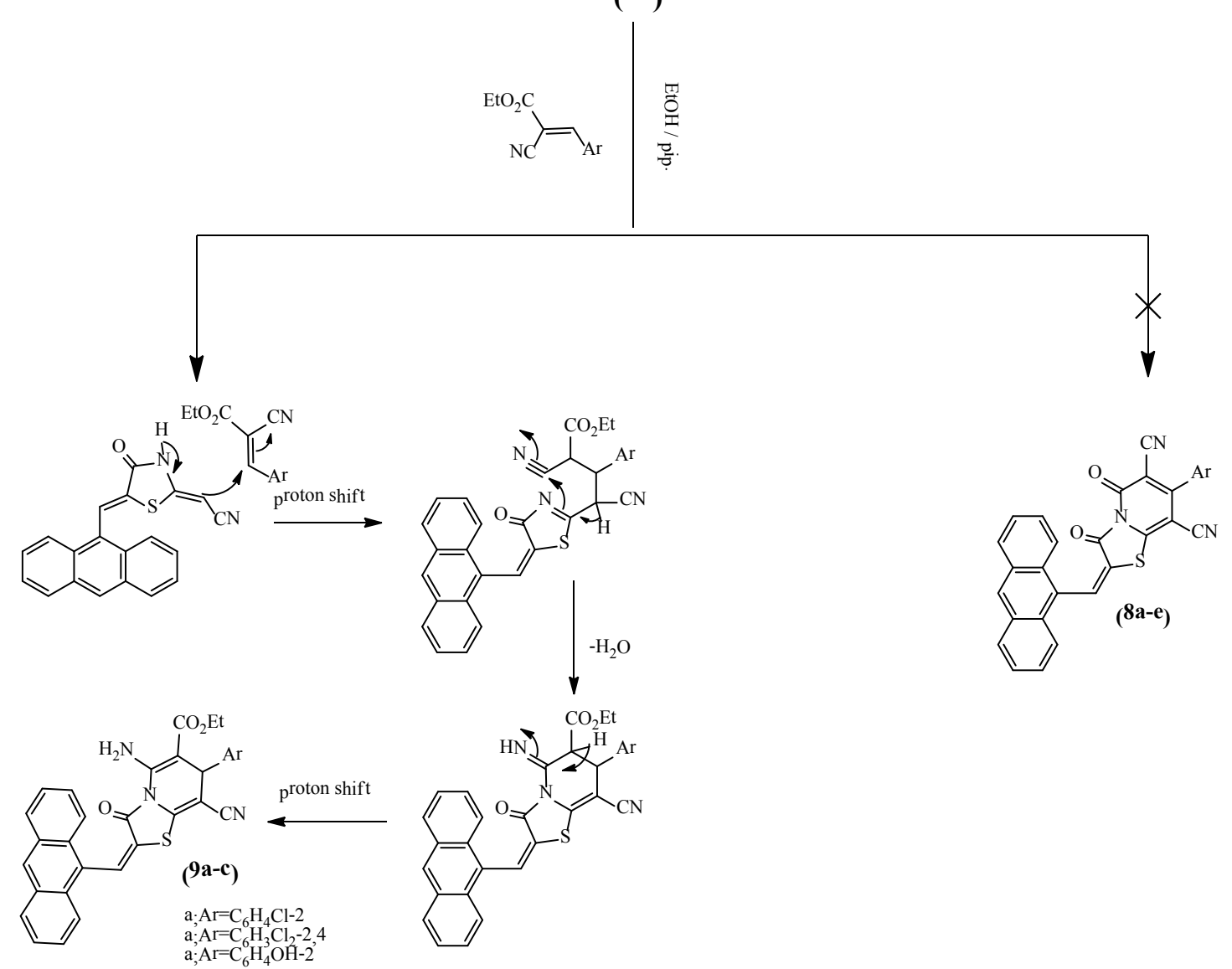

Scheme3<smiles>N#CC1=C(SCCC(=O)O)S/C(=C\c2c3ccccc3cc3ccccc23)C(=O)N2c3nc[nH]c(=O)c3C(c3ccc(Cl)cc3)C12</smiles>

(10)

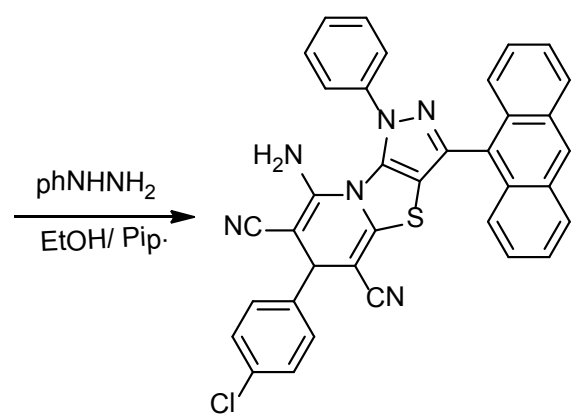

(11)

Scheme4

coccus aureus RCMB 010027, Staphylococcus epidermidis RCMB 010024 and BacillissubtilisRCMB 010063:; three Gram-negative bacteria, Neisseria gonorrhoeae RCMB 010079, Esherichia coli RCMB 010052 and KlebsiellapneumoniaeRCMB 010093. They were also evaluated for their in vitro antifungal potential against the following strains Aspergillus fumigatusRCMB 02564, Aspergillus-clavatusRCMB 02593 and GeotricumCandidumRCMB 05096, and the results were depicted in Table 1, 2. Antimicrobial tests were carried out by the agar well diffusion method using $(1 \mathrm{mg} / \mathrm{ml})$ in dimethyl sulfoxide
(DMSO) [33]. The inoculated plates were then incubated for $24 \mathrm{~h}$ at $37^{\circ} \mathrm{C}$. Ampicillin, Gentamycin and Amphotericin $\mathrm{B}(1 \mathrm{mg} / \mathrm{mL})$ were used as standard references for Gram positive bacteria, Gram negative bacteria and antifungal activity, respectively. After incubation time, antimicrobial activity was evaluated by measuring the inhibition zone diameters against the test organisms and compared with standard zone size ranges that determine susceptibility, intermediate susceptibility, or resistance to the screened compounds. Visual bacterial growth is observed only in areas in which the drug concentrations 
Synthesis, characterization and antimicrobial evaluation

are below those required for growth inhibition. The experiment was carried out in triplicate and the average zone of inhibition was calculated.In general, most of the tested compounds revealed better activity against the Gram-positive rather than the Gram-negative bacteria. Regarding to the structure activityrelationship of the thiazolidinones against Gram-positive bacteria and Gram-negative bacteria, the results revealed that compounds $2 \mathrm{~b}(\mathrm{MIC}=7.81,3.9,1.95 \mu \mathrm{g} \mathrm{mL}-$ 1), $4 \mathrm{a} \quad(\mathrm{MIC}=15.63,62.5,7.81 \mu \mathrm{g} \mathrm{mL}-1), 4 \mathrm{~b}$ $(\mathrm{MIC}=3.9,7.81,15.63, \mu \mathrm{g} \mathrm{mL}-1), 5 \quad(\mathrm{MIC}=$ $15.63,7.81,1.95,3.9, \mu \mathrm{g} \mathrm{mL}-1)$ and $6 \mathrm{~b}(\mathrm{MIC}=$ $1.95,0.98,7.81,1.95, \mu \mathrm{g} \mathrm{mL}-1)$ exhibited good spectrum antibacterial profile against the tested organisms. Compounds $2 b$ which contain ester group recorded higher activity than $2 \mathrm{a}$ which contain cyano group. Compounds $4 \mathrm{a}$ and $4 \mathrm{~b}$ which contain 4-chlorophenyl or 2-hydroxyphenyl respectively, showed equal activity against the tests organisms. Also, the equal activity was observed for compounds 4 and 5 which differ in the arylidene positions. Furthermore, meanwhile compound thiazolo[3,2-a]pyridine $6 \mathrm{~b}$ which contain 2-chlorophenyl revealed the highest activity, compound (6c) which contain dichlorophenyl does not showed any activity towards the tested organisms. On the other hand, compounds $2 \mathrm{a}, 6 \mathrm{c}, 9 \mathrm{a}, 9 \mathrm{~b}, 10$ and 11 exhibited weak to moderate growth inhibitory activity against the tested bacteria. Among these compounds $6 \mathrm{c}, 7$ and $9 \mathrm{~b}$ do not have any activity. The Gram-negative strain Neisseria gonorrhoeaeRCMB 010079 not affected with any of the tested compounds.

Table 1;Antibacterial activity of the synthesized compounds

\begin{tabular}{|c|c|c|c|c|c|c|}
\hline & \multicolumn{2}{|c|}{ Gram Positive Bacteria } & \multicolumn{4}{|c|}{ Gram Negative Bacteria } \\
\hline & S.aureus & S.epidermidis & B.subtilits & N.gonorrhoeae & E.coli & K.pneumoniae \\
\hline $2 \mathrm{a}$ & $0.58 \pm 15.2$ & $0.63 \pm 17.2$ & $0.42 \pm 19.2$ & $\mathrm{NA}$ & $0.72 \pm 15.6$ & $0.63 \pm 16.2$ \\
\hline $2 b$ & $0.25 \pm 18.2$ & $0.58 \pm 19.6$ & $0.63 \pm 22.4$ & NA & $0.72 \pm 19.2$ & $0.63 \pm 20.6$ \\
\hline $4 a$ & $0.72 \pm 16.7$ & $1.2 \pm 18.2$ & $0.43 \pm 20.3$ & NA & $1.2 \pm 18.9$ & $0.81 \pm 20.1$ \\
\hline $4 \mathrm{~b}$ & $0.58 \pm 19.4$ & $0.63 \pm 18.3$ & $0.63 \pm 20.3$ & NA & $1.2 \pm 17.3$ & $2.1 \pm 20.6$ \\
\hline 5 & $0.72 \pm 18.2$ & $0.44 \pm 13.3$ & $0.63 \pm 19.2$ & NA & $0.72 \pm 18.9$ & $1.2 \pm 20.3$ \\
\hline $6 b$ & $0.63 \pm 21.1$ & $0.72 \pm 21.9$ & $0.53 \pm 22.3$ & NA & $0.72 \pm 18.3$ & $0.25 \pm 22.4$ \\
\hline $6 c$ & NA & NA & NA & NA & NA & NA \\
\hline 7 & NA & NA & NA & NA & NA & NA \\
\hline $9 \mathrm{a}$ & $0.72 \pm 17.2$ & $0.72 \pm 12.4$ & $0.67 \pm 17.3$ & NA & $0.72 \pm 17.2$ & $0.58 \pm 17.9$ \\
\hline $9 b$ & NA & NA & NA & NA & NA & NA \\
\hline 10 & $0.63 \pm 11.6$ & $0.72 \pm 13.2$ & $0.42 \pm 14.6$ & NA & $0.58 \pm 13.1$ & $1.2 \pm 16.2$ \\
\hline 11 & $0.43 \pm 11.9$ & $0.43 \pm 11.2$ & $0.63 \pm 13.2$ & NA & $0.73 \pm 11.4$ & $1.2 \pm 13.3$ \\
\hline .St & $0.14 \pm 28.9$ & $0.18 \pm 25.4$ & $0.35 \pm 34.6$ & $0.58 \pm 22.3$ & $0.3 \pm 23.4$ & $0.15 \pm 26.3$ \\
\hline
\end{tabular}

Table 2; Antifungal activity of the synthesized compounds

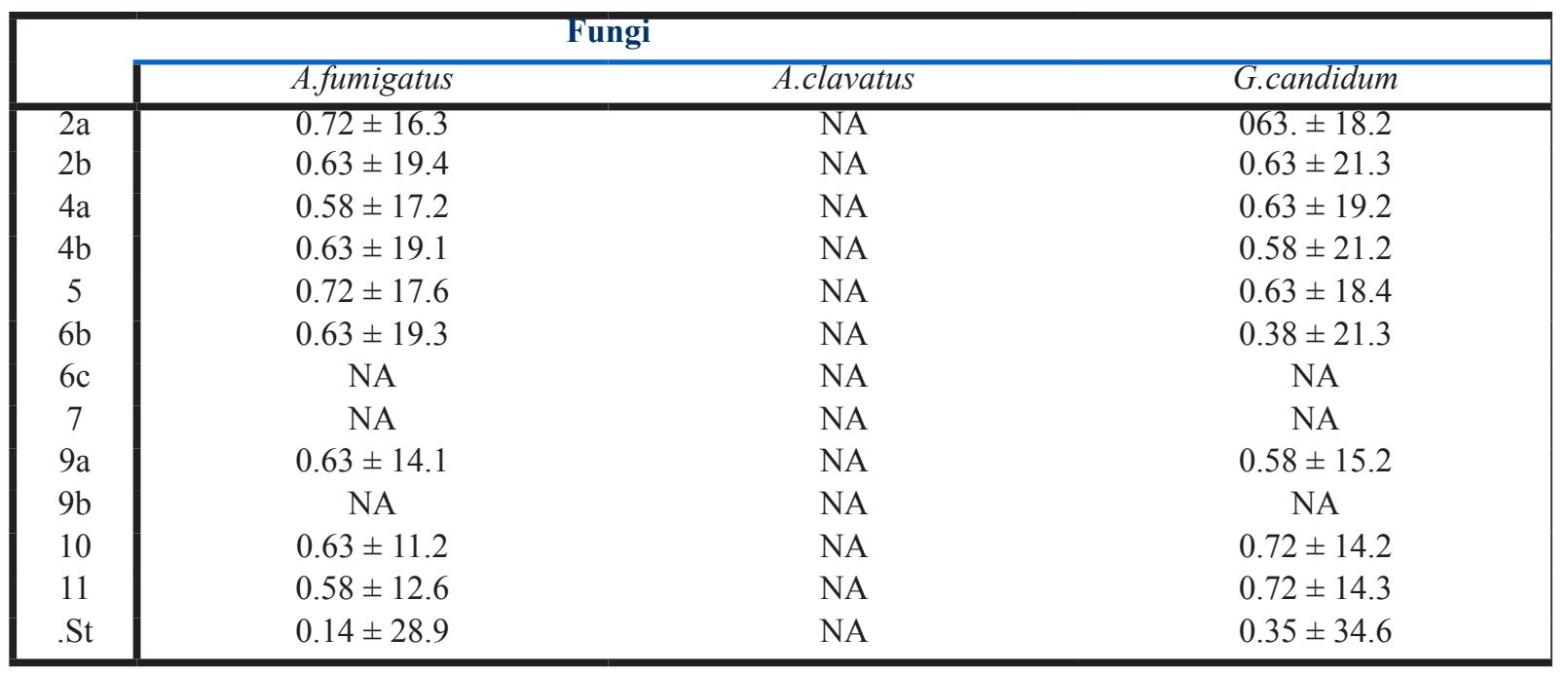


Regarding the activity of the tested compounds against the antifungal strains, the results revealed that compounds $2 \mathrm{~b}(\mathrm{MIC}=3.9,1.95 \mu \mathrm{g} \mathrm{mL}-1), 4 \mathrm{~b}$ $(\mathrm{MIC}=3.9,1.95, \mu \mathrm{g} \mathrm{mL}-1)$ and $6 \mathrm{~b}(\mathrm{MIC}=3.9$, $1.95, \mu \mathrm{g} \mathrm{mL}-1)$ proved to be equipotent to Amphotericin against Aspergillus fumigates (RCMB 02564), and GeotricumCandidum (RCMB 05096). On the other hand, none of the tested compounds showed any activity towards the strain Aspergillusclavatus (RCMB 02593). While, compounds 2a, 6c, 9a, 9b, 10 and 11 exhibited weak to moderate growth inhibitory activity against the tested strains.

\section{Conclusions}

We have described herein an efficient and convenient synthesis of some thiazolidinoe and thiazolopyridine derivatives. Twelve of the prepared compounds evaluated for their in vitro antibacterial and antifungal activities. The best antimicrobial activity was observed for 5-Amino-2-(anthracen9-ylmethylene)-7-(2-chlorophenyl)-3-oxo-3,7di-hydro-2H-thiazolo [3,2-a] pyrid- ine-6,8-dicarbonitrile (6b)followed by 2-[5-(anthra-cen9-yl-methylene)-4-oxothiazolidin-2-yl)]-3-(2-hydroxyphenyl)acryonitrile (4b).

Antimicrobial Activity as MICS $(\mu \mathrm{g} / \mathrm{ml})$ of tested samples against tested microorganisms:

\section{1EXPERIMENTAL}

Melting points are uncorrected.IR spectra were recorded on a Shimadzu 440 infrared spectrophotometer $(v$; cm-1) using the $\mathrm{KBr}$ technique (Shimadzu, Japan). 1HNMR spectra were recorded on a Varian Gemini spectrometer $(\delta$; ppm) $200 \mathrm{MHz}$ using TMS as internal standard. Mass spectra were recorded on a Jeol-JMS-600 mass spectrometer. Micro analytical data were obtained from the Micro analytical Research Centre, Faculty of Science, Cairo University.

2-[5-(Anthracen-9-ylmethylene)-4-oxothiazolidin-2-ylidene)acetonitrile (2a) and ethyl 2-[ 5-(anthracen-9-ylmethylene)-4-oxothiazolidin2-ylidene)acetate (2b)

\section{General procedure}

A mixture of anthraaldehyde $(0.01 \mathrm{~mol})$ was heated under reflux for $2 \mathrm{~h}$ with either $1 \mathrm{a}$ or $1 \mathrm{~b}$ $(0.01 \mathrm{~mol})$ in absolute ethanol $(20 \mathrm{~mL})$ catalyzed with piperidine. The solid product formed was collected by filtration and recrystallized from ethanol.

2-[5-(Anthracen-9-ylmethylene)-4-oxothiazolidin-2-ylidene)acetonitrile(2a).

Browns crystals; Mp $125-27^{\circ} \mathrm{C}$ (72\% yield).

\begin{tabular}{|c|c|c|c|c|c|c|}
\hline Sample & $2 b$ & $4 \mathrm{a}$ & $4 b$ & 5 & $6 b$ & \\
\hline microorganisms & \multicolumn{5}{|c|}{ (Minimum inhibitory concentration $(\mu \mathrm{g} / \mathrm{ml}$} & \\
\hline \multirow{2}{*}{$\begin{array}{c}\qquad \underline{\text { FUNGI }} \\
\text { Aspergillusfumigatus (RCMB 02564) }\end{array}$} & \multirow[b]{2}{*}{3.9} & \multirow[b]{2}{*}{15.63} & \multirow[b]{2}{*}{3.9} & \multirow[b]{2}{*}{7.81} & \multirow[b]{2}{*}{3.9} & $\begin{array}{c}\text { Amphotericin } \\
\mathrm{B} \\
\end{array}$ \\
\hline & & & & & & 0.98 \\
\hline Aspergillusclavatus (RCMB 02593) & NA & NA & NA & NA & NA & 1.95 \\
\hline $\begin{array}{l}\text { Geotricumcandidum (RCMB } \\
\text { 05096) }\end{array}$ & 1.95 & 3.9 & 1.95 & 7.81 & 1.95 & 0.49 \\
\hline \multirow{2}{*}{$\begin{array}{l}\text { Gram Positive Bacteria: } \\
\text { Staphylococcus aureus (RCMB } \\
\text { 010027) }\end{array}$} & \multirow[b]{2}{*}{7.81} & \multirow[b]{2}{*}{15.63} & \multirow[b]{2}{*}{3.9} & \multirow[b]{2}{*}{15.63} & \multirow[b]{2}{*}{1.95} & Ampicillin \\
\hline & & & & & & 0.49 \\
\hline $\begin{array}{l}\text { Staphylococcusepidermidis(RCMB } \\
010024)\end{array}$ & 3.9 & 62.5 & 7.81 & 7.81 & 0.98 & 0.49 \\
\hline Bacillissubtilis(RCMB 010063) & 1.95 & 15.63 & 3.9 & 1.95 & 0.98 & 0.24 \\
\hline \multirow{2}{*}{$\begin{array}{l}\text { Gram negativeBacteria: } \\
\text { Neisseria gonorrhoeae(RCMB } \\
010079 \text { ) }\end{array}$} & \multirow[b]{2}{*}{ NA } & \multirow[b]{2}{*}{ NA } & \multirow[b]{2}{*}{ NA } & \multirow[b]{2}{*}{ NA } & \multirow[b]{2}{*}{ NA } & Gentamycin \\
\hline & & & & & & 0.98 \\
\hline $\begin{array}{l}\text { Escherichia coli } \\
010052)\end{array}$ & 3.9 & 15.63 & 7.81 & 3.9 & 7.81 & 1.95 \\
\hline $\begin{array}{l}\text { Klebsiellapneumoniae } \\
\text { 0010093) }\end{array}$ & 3.9 & 7.81 & 1.95 & 3.9 & 1.95 & 0.49 \\
\hline
\end{tabular}


${ }^{1} \mathrm{H}$ NMR (DMSO-d6) $\delta /$ ppm5.16 (s, 1H, methine-H), 7.51-8.75 (m, 10H,Ar-H+methine-H) and $11.49\left(\mathrm{~s}, 1 \mathrm{H}, \mathrm{NH}\right.$, cancelled with $\left.\mathrm{D}_{2} \mathrm{O}\right)$; IR $(\mathrm{KBr}) \mathrm{cm}^{-1}:=3183(\mathrm{NH}), 3055(\mathrm{CH}-a r o m$.$) ,$ 2923 (CH-aliph.), 2195(C $\equiv \mathrm{N})$ and $1707(\mathrm{C}=\mathrm{O}$ thiazolidinone) ; Anal.Calcd.for $\mathrm{C}_{20} \mathrm{H}_{12} \mathrm{~N}_{2} \mathrm{O}_{\mathrm{S}}$ (328.07): C; 73.15, H; 3.68; N; 8.53 Found: $\mathrm{C}$; $73.52 \mathrm{H} ; 3.81, \mathrm{~N} ; 8.85$.

Ethyl2-[5-(anthracen-9-ylmethylene)4-oxothiazolidin-2-ylidene) acetate (2b).Yellows crystals; Mp $245-47^{\circ} \mathrm{C}$ (77 \% yield). ${ }^{1} \mathrm{H}$ NMR (DMSO-d6) $\delta /$ ppm $1.08\left(\mathrm{t}, 3 \mathrm{H}, \mathrm{CH}_{3}\right.$ ), 3.96(q, 2H, $\left.\mathrm{CH}_{2}\right), 5.57$ (s,1H, methine-H), 7.498.74(m, 9H,Ar-H), $9.01(\mathrm{~s}, 1 \mathrm{H}$, methine-H) and 11.49 (s, $1 \mathrm{H}, \mathrm{NH}$, exchangeable with $\left.\mathrm{D}_{2} \mathrm{O}\right)$; IR $(\mathrm{KBr}): \mathrm{cm}-1=3246(\mathrm{NH}), 3008(\mathrm{CH}-$ arom. $)$, 2892 (CH-aliph.) and $1689(2 \mathrm{C}=\mathrm{O}$ thiazolidinone and ester ); Anal. Calcd.for $\mathrm{C}_{22} \mathrm{H}_{17} \mathrm{NO}_{3} \mathrm{~S}$ (375.09): C; 70.38, H; 4.56; N; 3.73. Found: C; $70.52 \mathrm{H} ; 4.81, \mathrm{~N} ; 3.52$.

2-(Anthracen-9-yl)-2-(4-oxothiazolidin2-ylidene) acetonitrile (3)

To a solution of 2-(anthracene-9-yl-methylene) malononitrile $(0.01 \mathrm{~mol})$ in acetic acid $(20 \mathrm{~mL})$, thioglycollic acid $(0.01 \mathrm{~mol})$ was added. The solution was heated under reflux for $2 \mathrm{~h}$. The solid product formed was collected by filtration and recrystallized from ethanol.

Yellow powder;Mp 160-62 ${ }^{\circ} \mathrm{C}$ (54 \%yield). IR (KBr): $\mathrm{cm}^{-1}=3323(\mathrm{NH}), 3049(\mathrm{CH}$-arom.), 2929 (CH-aliph.), 2193( $(\mathrm{C} \equiv \mathrm{N}))$ and $1653(\mathrm{C}=\mathrm{O}$ thiazolidinone); Anal.Calcd. for $\mathrm{C}_{20} \mathrm{H}_{12} \mathrm{~N}_{2} \mathrm{OS}$ (328.07): C; 73.15, H; 3.68; N; 8.53 Found C; $73.61 \mathrm{H} ; 3.25, \mathrm{~N} ; 8.31$.

2-[5-(Anthracen-9-yl-methylene)-4-oxo-4,5dihydrothiazolidin-2-yl)]-3-aryl-acrylonitrile $(4 a-c)$.

\section{General procedure}

A mixture of 4-thiazolidinone derivative $2 \mathrm{a}$ $(0.01 \mathrm{~mol})$ and aromatic aldehydes $(0.01 \mathrm{~mol})$, in absolute ethanol $(20 \mathrm{~mL})$ catalyzed with piperidine was heated under reflux for $3 \mathrm{~h}$. The solid product formed was collected by filtration and recrystallized from ethanol.

2-[5-(Anthracen-9-yl-methylene)-4-oxo-4,5dihydrothiazolidinin-2-yl)]-3-(4-chlo- ro- phe- nyl ) acrylonitrile (4a).

Reddish brown crystals; Mp $150-52{ }^{\circ} \mathrm{C}(67$ \%yield). ${ }^{1} \mathrm{HNMR}$ (DMSO-d6) $\delta / \mathrm{ppm}$ 6.58-8.66 (m,13H,Ar-H), 9.01,9.05 (2s, 2H, methine-H); IR (KBr): $\mathrm{cm}^{-1}=3056(\mathrm{CH}$-arom.), $2978(\mathrm{CH}-$ aliph.), 2195(C $\equiv \mathrm{N})$ and $1709(\mathrm{C}=\mathrm{O}$ thiazolidinone) ;Anal. Calcd.for $\mathrm{C}_{27} \mathrm{H}_{15} \mathrm{ClN}_{2} \mathrm{OS}(450.94)$ C; 71.91, H; 3.35; N; 6.21 Found: C; $72.01 \mathrm{H}$; $3.89, \mathrm{~N} ; 6.19$.

2-[5-(anthracen-9-yl-methylene)-4-oxothiazolidin-2-yl)]-3-(2-hydroxyphenyl)-acrylonitrile (4b).

Brown crystals; Mp 130-32 ${ }^{\circ} \mathrm{C}$ (78 \% yield). IR (KBr) cm-1= 3330 (br, OH), 3055(CHarom. $), 2204(\mathrm{C} \equiv \mathrm{N})$ and $1655(\mathrm{C}=\mathrm{O}$ thiazolidinone); $\mathrm{MS} \mathrm{m} / \mathrm{z}(\%)$ : $432(\mathrm{M}+, 0.76)$; Anal. Calcd.for $\mathrm{C}_{27} \mathrm{H}_{16} \mathrm{~N}_{2} \mathrm{O}_{2} \mathrm{~S}$ (432.09) $\mathrm{C} ; 74.98, \mathrm{H}$; 3.73; N; 6.48 Found: C; 74.62 H; 3.98, N; 6.19.

2-[5-(Anthracen-9-yl-methylene)-4-oxo-4,5dihydrothiazolidin-2-yl)]-3-(2-hydroxy-naphthalen-1-yl)acrylonitrile (4c)

Brown crystals; Mp $\quad 160-62 \quad{ }^{\circ} \mathrm{C} \quad(67 \%$ yield). ${ }^{1} \mathrm{HNMR}$ (DMSO-d6) $\delta / \mathrm{ppm} \quad 6.98-8.21$ (m,15H,Ar-H), 9.01,9.04 (2s, 2H, methine-H), (s, 1H,OH); IR (KBr) : $\mathrm{cm}^{-1}=3221$ (br. OH), $2205(\mathrm{C} \equiv \mathrm{N})$ and $1651 \quad(\mathrm{C}=\mathrm{O}$ thiazolidinone); Anal. Calcd.for $\mathrm{C}_{31} \mathrm{H}_{18} \mathrm{~N}_{2} \mathrm{O}_{2} \mathrm{~S}$ (482.11) C; 77.16, $\mathrm{H}$; 3.76; N; 5.81 Found: C; 77.32; H; 3.52, N; 5.41 .

3-(Anthracen-9-yl)-2-(5-(arylmethylidine)4-oxo-4, 5-dihydrothiazol-2-yl) acrylo- nitriles $(5 \mathrm{a}, \mathrm{b})$

To a solution of 4-thiazolidinone derivative $3(0.01 \mathrm{~mol})$ in absolute ethanol $(20 \mathrm{~mL})$ catalyzed with piperidine either $\alpha$-hdroxynaphthaldehyde or p-chlorobenz-aldehyde $(0.01 \mathrm{~mol})$ was added. The solution was heated under reflux for $3 \mathrm{~h}$. The solid product formed was collected by filtrationrecrystallized from ethanol.

3-(Anthracen-9-yl)-2-(5-(2-hydroxynaphthylidine) -4-oxo-4,5-dihydrothiazol-2-yl) acrylonitrile (5a)

Brown crystals,Mp $160-62{ }^{\circ} \mathrm{C}$ (67 \%yield). IR $(\mathrm{KBr}) \quad: \mathrm{cm}^{-1}=3375($ br. $\mathrm{OH}), 3052(\mathrm{CH}-$ arom.),2927(CH-aliph), 2206(C $\equiv \mathrm{N})$ and 1662 $(\mathrm{C}=\mathrm{O}) ; \mathrm{MS} \mathrm{m} / \mathrm{z}(\%): 481$ (M-1), (56.70); Anal. 
Calcd.for C31H18N2O2S (482.11): C; 77.16, H; 3.76; N; 5.81 Found: C; 77.65; H; 3.92, N; 5.98.

3-(Anthracen-9-yl)-2-(5-(4-chlorobenzylidene) -4-oxo-4,5-dihydrothiazol-2-yl) acrylonitrile (5b)

Brown crystals; $\mathrm{Mp} \quad 100-02^{\circ} \mathrm{C} \quad(75 \%$ yield). ${ }^{1} \mathrm{HNMR}$ (DMSO-d6) $\delta / \mathrm{ppm} \quad 6.59-8.10$ (m, 13H, Ar-H), 8.20, 8.99 (2s, 2H, methine-H) ; IR (KBr)cm ${ }^{-1}:=3056$ (CH-arom.), 2927 (CHaliph), $2198(\mathrm{C} \equiv \mathrm{N})$ and $1658(\mathrm{C}=\mathrm{O}$ thiazolidinone ) ; Anal. Calcd for $\mathrm{C}_{27} \mathrm{H}_{15} \mathrm{~N}_{2} \mathrm{ClOS}$ (450.94) C; 71.91, H; 3.35; N; 6.21 Found: C; $72.01 \mathrm{H}$; $3.79, \mathrm{~N} ; 6.10$.

5-Amino-2-(anthracen-9-ylmethylene)-7aryl-3-oxo-3,7-dihydro-2H-thiazolo[3,2-a] pyridine-6,8-dicarbonitriles (6a-d)

A mixture of 4-thiazolidinone derivative $2 \mathrm{a}(0.01 \mathrm{~mol})$ and $\alpha$-cyanocinnamonitrile $(0.01 \mathrm{~mol})$, in absolute ethanol $(20 \mathrm{~mL})$ catalyzed with piperidine was heated under reflux for $6 \mathrm{~h}$. The solid product formed was collected by filtration and recrystallized from ethanol.

5-Amino-2-(anthracen-9-ylmethylene)-7-(4chlorophenyl)-3-oxo-3,7-dihydro-2H-thiazolo [3,2-a] pyridine-6,8-dicarbonitrile $(6 \mathrm{a})$

Brown powder,Mp $160-62^{\circ} \mathrm{C}$ (73\%yield). ${ }^{1} \mathrm{H}$

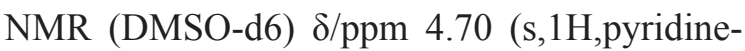
$\mathrm{H})$, 6.37-8.80 (m, $16 \mathrm{H}, \mathrm{Ar}-\mathrm{H}+$ methine- $\mathrm{H}+\mathrm{NH}_{2}$; exchangeable with $\left.\mathrm{D}_{2} \mathrm{O}\right) ; \mathrm{IR}(\mathrm{KBr}) \mathrm{cm}^{-1}$ : $=3331$, $3354\left(\mathrm{NH}_{2}\right), 3051$ (CH-arom.) , $2200(\mathrm{C} \equiv \mathrm{N})$ and $1708(\mathrm{C}=\mathrm{O}$ thiazolidinone $) ; \mathrm{MS} \mathrm{m} / \mathrm{z}(\%)$ : 516, (M+, 6.89); Anal. Calcd.for $\mathrm{C}_{30} \mathrm{H}_{17} \mathrm{ClN}_{4} \mathrm{OS}$ (517.00) C; 69.69, H; 3.31; N; 10.84 Found: C; 70.12, H; 3.64, N; 10.37 .

5-Amino-2-(anthracen-9-ylmethylene)7-(2-chlorophenyl)-3-oxo-3,7-dihydro-2Hthiazolo[3,2-a]pyridine-6,8-dicarbonitrile (6b)

Yellow powder;M.p295-97\% $\mathrm{C}$ (67 \%yield); ${ }^{1} \mathrm{H}$ NMR (DMSO-d6) $\delta / p p m 5.09$ (s, 1H,pyridine$\mathrm{H})$, 7.35-7.98 (m,16H,Ar-H + methine- $\mathrm{H}+\mathrm{NH}_{2}$; exchangeable with $\left.\mathrm{D}_{2} \mathrm{O}\right)$; IR $(\mathrm{KBr}): \mathrm{cm}^{-1}:=$ 3388,3286 ( $\left.\mathrm{NH}_{2}\right), 3050(\mathrm{CH}$-arom.) $2197(\mathrm{C} \equiv \mathrm{N})$ and $1715(\mathrm{C}=\mathrm{O}$ thiazolidinone);Anal. Calcd.for $\mathrm{C}_{30} \mathrm{H}_{17} \mathrm{Cl} \mathrm{N} \mathrm{N}_{4} \mathrm{OS}(517.00) \mathrm{C} ; 69.69, \mathrm{H} ; 3.31 ; \mathrm{N}$; 10.84 Found: C; 69.87, H; 3.04, N; 10.63 .

5-Amino-2-(anthracen-9-ylmethylene)- 7-(2,4-dichlorophenyl) -3-oxo-3,7-dihydro-2Hthiazolo [3,2-a] pyridine-6,8-dicarbonitrile (6c)

Yellow powder; Mp 290-92 $2^{\circ} \mathrm{C}$ (61\%yield). ${ }^{1} \mathrm{H}$ NMR (DMSO-d6) $\delta /$ ppm5.10(s,1H,pyridine$\mathrm{H})$, 7.55-7.91 (m, $15 \mathrm{H}, \mathrm{Ar}-\mathrm{H}+$ methine- $\mathrm{H}+\mathrm{NH}_{2}$; exchangeable with $\left.\mathrm{D}_{2} \mathrm{O}\right)$; IR $(\mathrm{KBr}, \mathrm{cm}-1)$ : = 3384,3289 $\left(\mathrm{NH}_{2}\right), 3055(\mathrm{CH}$-arom.) $2198(\mathrm{C} \equiv \mathrm{N})$ and $1721(\mathrm{C}=\mathrm{O}$ thiazolidinone $)$; Anal.Calcd.for $\mathrm{C}_{30} \mathrm{H}_{16} \mathrm{C}_{12} \mathrm{~N}_{4} \mathrm{OS}(551.45) \mathrm{C} ; 65.34, \mathrm{H} ; 2.92 ; \mathrm{N}$; 10.16 Found: C; 65.72, H; 3.23, N; 10.42 .

5-Amino-2-(anthracen-9-ylmethylene)-7(2,4-anthracen-9-yl)-3-oxo-3,7-dihydro-2Hthiazolo[3,2-a]pyridine-6,8-dicarbonitrile (6d)

Brown powder; Mp170-72 ${ }^{\circ} \mathrm{C}$ (61\%yield) ${ }^{1} \mathrm{H}$ NMR (DMSO-d6) $\delta /$ ppm5.10 (s,1H,pyridine$\mathrm{H})$, 7.55-7.91 (m, 21H,Ar-H + methine- $\mathrm{H}+$; $\mathrm{NH}_{2}$ exchangeable with $\left.\mathrm{D}_{2} \mathrm{O}\right)$; IR $(\mathrm{KBr}): \mathrm{cm}^{-1}$ $=3332,3207\left(\mathrm{NH}_{2}\right), 3051$ (CH-arom.), 2006 $(\mathrm{C} \equiv \mathrm{N})$ and $1707(\mathrm{C}=\mathrm{O}$ thiazolidinone $)$; Anal. Calcd for $\mathrm{C}_{38} \mathrm{H}_{22} \mathrm{~N}_{4} \mathrm{OS}(582.15) \mathrm{C} ; 78.33, \mathrm{H}$; 3,81; N; 9.62 Found: C; 78.73, H; 3.21, N; 9.13.

3,9-Diamino-4-(anthracen-9-yl)-7-(4hydroxyphenyl)-4,7-dihydropyrano[2',3':4,5] thiazolo [3,2-a]pyridine-2,6,8-tricarbonitrile (7)

To a solution of 4-thiazolidinone derivative $2 \mathrm{a}$ $(0.01 \mathrm{~mol})$ in absolute ethanol $(20 \mathrm{~mL})$ catalyzed with piperidine, p-hydroxybenzaldehyde $(0.01 \mathrm{~mol})$ and malononitrile $(0.02 \mathrm{~mol})(1: 1: 2$ moalr ratio) were added. The reaction mixture was heated under reflux for $6 \mathrm{~h}$. The solid product formed was collected by filtration and recrystallized from ethanol.

3,9-Diamino-4-(anthracen-9-y1)-7-(4hydroxyphenyl)-4,7-dihydropyrano[2',3':4,5] thiazolo[3,2-a]pyridine-2,6,8-tricarbonitrile (7)

Yellow powder; Mp $140-42^{\circ} \mathrm{C}$ (58\%yield); ${ }^{1} \mathrm{H}$ NMR (DMSO-d6) $\delta / p p m ~ 4.14$ (s, 1H, pyran-H), 4.34 (s, $1 \mathrm{H}$, pyridine- $\mathrm{H}), 6.36-8.20(\mathrm{~m}$, $17 \mathrm{H}, \mathrm{Ar}-\mathrm{H}+2 \mathrm{NH}_{2}$; exchangeable with $\mathrm{D} 2 \mathrm{O}$ ) and 10.34 (br, $1 \mathrm{H}, \mathrm{OH}$; exchangeable with $\mathrm{D} 2 \mathrm{O}$ ); IR $(\mathrm{KBr}): \mathrm{cm}^{-1}=3316,3206$ ( broad NH2), and 2201(C $\equiv \mathrm{N}) ; \mathrm{MS} \mathrm{m} / \mathrm{z}(\%): 564(\mathrm{M}+, 41.47)$; Anal. Calcd.for $\mathrm{C}_{33} \mathrm{H}_{20} \mathrm{~N}_{6} \mathrm{O}_{2} \mathrm{~S}$ (564.14) C; 70.20, $\mathrm{H}$; 3.57; N; 14.88 Found: C; 69.83, H; 3.33, N; 14.25 .

Ethyl-5-amino-2- anthracen-9-ylmethylene)- 
7aryl-8-cyano-3-oxo-3,7-dihydro-2H - t hiazolo [3,2-a] pyridine-6-carboxylate (9a-c)

A mixture of 4-thiazolidinone derivative $2 \mathrm{a}(0.01 \mathrm{~mol})$, and $\alpha$-cyanocinnamonitriles $(0.01 \mathrm{~mol})$, in absolute ethanol $(20 \mathrm{~mL})$ catalyzed with piperidine was heated under reflux for $6 \mathrm{~h}$. The solid product formed was collected by filtration and recrystallized from ethanol.

Ethyl-5-amino-2-(anthracen-9-ylmethylene)7-(2-chlorophenyl)-8-cyano-3-oxo-3,7-dihydro$2 \mathrm{H}$-thiazolo $[3,2-\mathrm{a}]$ pyridine-6-carboxylate $(9 \mathrm{a})$.

Yellow powder; $\mathrm{Mp} 255-57{ }^{\circ} \mathrm{C}$ (67\%yield). ${ }^{1} \mathrm{H}$ NMR (DMSO-d6) $\delta / \mathrm{ppm} 0.83$ (t, J=7.5Hz, $\left.3 \mathrm{H}, \mathrm{CH}_{3}\right), 3.88\left(\mathrm{q}, \mathrm{J}=7.5 \mathrm{~Hz}, 2 \mathrm{H}, \mathrm{CH}_{2}\right)$, 5.16(s, $1 \mathrm{H}$, pyridine- $\mathrm{H}), 7.25-7.96(\mathrm{~m}, 16 \mathrm{H}, \mathrm{Ar}-$ $\mathrm{H}+$ methine- $\mathrm{H}+\mathrm{NH}_{2}$ exchangeable with $\left.\mathrm{D}_{2} \mathrm{O}\right)$; IR $(\mathrm{KBr}): \mathrm{cm}^{-1}=3414,3289\left(\mathrm{NH}_{2}\right), 2210(\mathrm{C} \equiv \mathrm{N})$ and $1714,1660(\mathrm{C}=\mathrm{O}$ ester and thiazolidinone); Anal. Calcd for $\mathrm{C}_{32} \mathrm{H}_{22} \mathrm{C}_{1} \mathrm{~N}_{3} \mathrm{O}_{3} \mathrm{~S}$ (563.50): C; 68 . 14, H; 3.93; N; 7.45 Found: C; 68.55, H; 3.65, $\mathrm{N} ; 7.13$.

Ethyl-5-amino-2-(anthracen-9-ylmethylene)8-cyano-7-(2,4-dichlorophenyl)-3-oxo-3,7-dihydro-2H -thiazolo[3,2-a]pyridine-6-carboxylate $(9 \mathrm{~b})$

Yellow powder; $\mathrm{Mp} 220-22^{\circ} \mathrm{C}$ (68\%yield). ${ }^{1} \mathrm{H}$ NMR (DMSO-d6) 0.92(t, J=7.5Hz, 3H, $\mathrm{CH}_{3}$ ), $3.89\left(\mathrm{q}, \quad \mathrm{J}=7.5 \mathrm{~Hz}, 2 \mathrm{H}, \mathrm{CH}_{2}\right), 5.10(\mathrm{~s}, 1 \mathrm{H}$, pyridine-H), 7.38-9.05 (m, 15H,Ar-H + methine-H ; $\mathrm{NH}_{2}$; exchangeable with $\left.\mathrm{D}_{2} \mathrm{O}\right)$; IR $(\mathrm{KBr}): \mathrm{cm}^{-1}=$ 3392,3276 (NH2), $2206(\mathrm{C} \equiv \mathrm{N})$ and 1713,1665 $(\mathrm{C}=\mathrm{O}$ ester and thiazolidinone $)$; Anal. Calcd for $\mathrm{C}_{32} \mathrm{H}_{21} \mathrm{C}_{12} \mathrm{~N}_{3} \mathrm{OS}(598.00) \mathrm{C} ; 64.22, \mathrm{H} ; 3.54$; $\mathrm{N} ; 7.02$ Found: C; 64.52, H; 3.23, N; 7.42.

Ethyl-5-amino-2-(anthracen-9-ylmethylene)8-cyano-7-(2-hydroxyphenyl)-3-oxo-3,7-dihydro-2H-thiazolo[3,2-a] pyridine-6-carboxylate $(9 \mathrm{c})$

Yellow powder; $\mathrm{Mp} 225-27^{\circ} \mathrm{C}$ (61\%yield). ${ }^{1} \mathrm{H}$ NMR (DMSO-d6) 1.09 (t, J=7.5Hz, 3H, $\left.\mathrm{CH}_{3}\right), 3.96\left(\mathrm{q}, \mathrm{J}=7.5 \mathrm{~Hz}, 2 \mathrm{H}, \mathrm{CH}_{2}\right), 5.56(\mathrm{~s}, 1 \mathrm{H}$, pyridine- $\mathrm{H}), 7.56-8.27(\mathrm{~m}, 17 \mathrm{H}, \mathrm{Ar}-\mathrm{H}+\mathrm{me}-$ thine- $\mathrm{H}+\mathrm{NH}_{2}$; exchangeable with $\left.\mathrm{D}_{2} \mathrm{O}\right), 12.20$ (br, $1 \mathrm{H}, \mathrm{OH}$; exchangeable with $\left.\mathrm{D}_{2} \mathrm{O}\right)$; IR $(\mathrm{KBr})$ : $\mathrm{cm}-1=3429,3289\left(\mathrm{NH}_{2}\right), 2198(\mathrm{C} \equiv \mathrm{N})$ and 1721 $(\mathrm{C}=$ Oester and thiazolidinone; Anal. Calcd.for
$\mathrm{C}_{32} \mathrm{H}_{23} \mathrm{~N}_{3} \mathrm{O}_{4} \mathrm{~S}$ (545.14): C; 70.44, H; 4.25; N; 7.70; Found: C; 70.11, H; 4.66, N; 7.12.

8 -(anthracen-9-ylmethylene) - 5-(4chlorophenyl)-4,9-dioxo-4,5,8,9-tetrahydro-3H -thiazolo[3',2':1,6]pyrido[2,3-d]pyrimidine6-carbonitrile (10)

A mixture of $2 \mathrm{a}(0.01 \mathrm{~mol})$ and formic acid $(0.01 \mathrm{~mol})$ was heated under reflux for $6 \mathrm{~h}$. The solid product formed was collected by filtration and recrystallized from ethanol.

8 -(anthracen-9-ylmethylene)-5-(4chlorophenyl)-4,9-dioxo-4,5,8,9-tetrahydro-3H -thiazolo[3',2':1,6]pyrido[2,3-d]pyrimidine6-carbonitrile (10)

Brown powder; Mp 225-27 ${ }^{\circ} \mathrm{C}$ (59\%yield. ${ }^{1} \mathrm{H}$ NMR (DMSO-d6), 4.97 (s,1H,pyridine-H), 5.20 (s,1H, pyrimidine-H),7.20-8.22 (m, 13H,Ar$\mathrm{H}), \quad 8.88(\mathrm{~s}, 1 \mathrm{H}$, methine-H), $9.64 \quad(\mathrm{~s}, 1 \mathrm{H}, \mathrm{NH}$; exchangeable with $\mathrm{D} 2 \mathrm{O})$; IR $(\mathrm{KBr})$ : $\mathrm{cm}^{-1}$ $=3181(\mathrm{NH}), 2227(\mathrm{C} \equiv \mathrm{N})$ and $1712(\mathrm{C}=\mathrm{O})$; Anal. Calcd. for $\mathrm{C}_{31} \mathrm{H}_{17} \mathrm{C}_{1} \mathrm{~N}_{4} \mathrm{O}_{2} \mathrm{~S}(545.50) \mathrm{C} ; 68.32, \mathrm{H}$; 3.14; N; 10.28 Found: C; 68.21, H; 2.98, N; 10.52 .

8 - amino-3-(anthracen-9-y1)-6-(4chlorophenyl)-1-phenyl-1,6-dihydropyrazolo[3',4': 4,5] thiazolo[3,2a]pyridine-5,7-dicarbonitrile (11)

To a solution of $2 \mathrm{a}(0.01 \mathrm{~mol})$ in absolute ethanol $(20 \mathrm{~mL})$, phenylhydrazine $(0.01 \mathrm{~mol})$ was added. The reaction mixture was heated under reflux for $6 \mathrm{~h}$. The solid product formed was collected by filtration and recrystallized from ethanol.

8 - a mino-3 - (anthracen-9-yl)- 6-(4chlorophenyl)-1-phenyl-1,6-dihydropyrazolo[3',4': 4,5] thiazolo[3,2a]pyridine-5,7-dicarbonitrile (11)

Brown powder, (56\%yield), Mp.190$92^{\circ} \mathrm{C}$. IR $(\mathrm{KBr}): \mathrm{cm}^{-1}=3432,3216\left(\mathrm{NH}_{2}\right)$ and $2211(\mathrm{C} \equiv \mathrm{N}) ; \mathrm{MS} \mathrm{m} / \mathrm{z}(\%): 604(\mathrm{M}+), 44.36)$; Anal.Calcd.for $\mathrm{C}_{36} \mathrm{H}_{23} \mathrm{C}_{1} \mathrm{~N}_{6} \mathrm{~S}$ (604.50) C; 71.40, $\mathrm{H}$; 3.50; N; 13.89, Found: C; 71.03, H; 3.71, N; 14.12

\section{REFERENCES}

[1] V. V. Mulwad, A. A. Mir, and H. T. parmar, Indian Journal of chemistry, 48B, 137(2009). 
[2] C. Sun, S .Ji , and Y . Liu, J. Chin. Chem. Soc.,55,292 (2008).

[3]S.Miwatashi, Y. Arikawa,E.Kotani, M. Miyamoto, K.I. Naruo, H. Kimura, T.Tanaka, S. Asahi andS .Ohkawa, J. Med. Chem.,48, 5966 (2005) .

[4] C. Papadopoulou,A.Geronikakiand D. Hadjipavlou. Litina,Il Farmaco,60, 969(2005).

[5] H. I. Ei-Subbagh and A. M Al-Obaid, Eur. J. Med. Chem.,31,1996, 1017.

[6] M. S.Sondhi, N. Singh, M.Johar and A. Kumar, Bioorg. Med. Chem;13,6158(2005)..

[7] W. W. Wardkhan, M. A. Yousef, F. I. Hameed and S. A. Ouf, Journal of the Chinese Chemical Society,55, 1133(2008).

[8]R. Pereira, C.Gaudon, B. Iglesias, P .Germain,H.Gronemeyer and A. R de Lera. Bioorg.Med. Chem; Lett,16, 49(2006)

[9]Y.Tsuruni, H. Ueda, K. Hayashi, S.Takase, M. Nishikawa, S.Okuhara andM.J.Kiyoto,Antibi ot;48,1066(1995).

[10] D. S.Millan, R. H. Prager, C. Brand and P. H.Hart, Tetrahedron,56,811(2000).

[11] W.L. Wang,D.Y.Yao,M.Gu, M. Z. Fan,J.Y. Li,Y.C. Xing and F.J. Nan, Bioorg.Med. Chem. Lett.,15, 5284(2005).

[12] J. Clough, S. Chen, E.M Gordon, C.Hackbarth, S. Lam,J.Trias, R. J.White, Q Candiani, S.Donadio,G. Romano, R.Ciabatti and J. W.Jacobs, Bioorg. Med. Lett;13,3409(2003).

[13] M. S.Chande and V. Suryanarayan, J. Chem. Res.,6,345(2005).

[14]S. K.Srivastava, R. Y. adav and S. D. Srivasta, J.IndianChem .Soc., 81, 342 (2004).

[15] S. S. Mishra, K. Srivastava, and S. D Srivasta, Indian J Chem., 36B, 826(1997).

[16] K. Poreba,A. Opolski,J. Wietrzyk and M. Kowalska, Arch. Pharm.,219334 (2001).

[17]K. Poreba, A. Opolski andJ.Wietrzyk, Acta Pol. Pharm.,59,215(2002) .

[18] K. Vira,S.Martin,W. KarePMilanand K. Jarmila, Il Farmaco,54, 666(1999).
[19] M. P. Marco,T.U . Daniela, G. P. Pietro and B. Z..P .Fabrizio, Il Farmaco,55,669(2000).

[20] B. P. Pregnolato, M. I .Gamb and A. G.Mellerio, J. Heterocyclic Chem.,301491(1993).

[21] G. M. Maria, F. Valeria,Z. Daniele, V.Luciano and B. Elena,IlFarmaco,56, 587(2001).

[22]M. E.azab, G. A. M. el-hag ali, and A.H. F. abdelwahab, Acta Pharm., 53, 213(2003). .

[23]M. S. A.El-gaby, G. A. M. El-hagali, A. A. AL-Maghraby, M. T.abd el-rahman, and M. H. HelaL, European Journal of medicinal chemistry ,44(10),414(2009).

[24]G. A. M. El-hag ali, R. Q. Lamphon , A. Khali and A. A. El- maghraby; Phosphorus, Sulfur, and Silicon,180(8),1909(2005).

[25]T. I., G. A. M. El-hag ali, A. Khalil, and A. A. A. El-adasy, Phosphorus, Sulfur, and Silicon; 180(1), 19(2005).

[26] G. A. M. El-hag ali, M. T. Abdelrahman, M. H. M. Helaland M. S. A. El-gaby, Phosphorus, Sulfur, and Silicon ; 183,3023(2008).

[27] M.H. Helal, S.A. El-Awdan , M.A. Salem, T.A. Abdelaziz , Y. A. Moahamed, A.A. El-Sherif, G.A. M. Mohamed,SpectrochimicaActa Part A: Molecular andBiomolecular Spectroscopy 135, 764(2015).

[28] H. Khamees, G. A.AbdelJaleel, Mahmed.E.Azab, Gameel.A.M. Mohamed ,Tarek.A.Abd-elaziz1, H..A.Eyada; J. Atoms and Molecules ,3(2),478(2013).

[29] K.U. Sadek, E.A. Hafez, A. E. Mourad, M.H. Elnagdy, Z. Naturforsch,39B, 824 (1984).

[30]M. Teresa Cocco , C. Congiu, V. Lilliuand V.Onnis, European Journal of Medicinal Chemistry 40,1365 (2005).

[31] W.J. Scott, J. Dumas, S. Boyer,W. Lee,Y. Chen, B. Phillips, s. verna,J. Chen, J.Fan, B. Raudenbush, L.Yi, Q. Zhu, L. Adnane, US Patent 0235829A1,2004.

[32] K. Tatsumi, T. Yamanuchi, K. Kiyono, K. Kishi, Y. Yanagihara,T. Imaoka, T. Kawaguchi, J. Biochem., (Tokyo) $114,912(1993)$.

[33] W. Hewitt and S. Vincent, Theory and Application of Microbiological Assay, Academic press, New York (1989). 